\title{
AUTOMATIC CALIBRATION SYSTEM FOR MICRO-DISPLACEMENT DEVICES
}

\author{
Hung-Ta Shih ${ }^{1}$, Yung-Cheng Wang ${ }^{2}$, Lih-Horng Shyu ${ }^{3}$, Pi-Cheng Tung ${ }^{1}$, Wen-Yuh Jywe ${ }^{4}$, Jia- \\ Hong Chen ${ }^{4}$ \\ ${ }^{1}$ Department of Mechanical Engineering, National Central University \\ ${ }^{2}$ Department of Mechanical Engineering, National Yunlin University of Science and \\ Technology \\ ${ }^{3}$ Department of Electro-Optical Engineering, National Formosa University \\ ${ }^{4}$ Department of Automation Engineering, National Formosa University
}

\begin{abstract}
With the industrial development and the advances in micro - displacement technology, the demands on piezo transducers are increasing. For piezo transducers, the error inspections of the non-linearity and the hysteresis are necessary procedure before piezo transducers utilized. Due to the possible decline or damage during the employment of the transducers, it is important to provide the automatic calibration system.

In this investigation, a self-developed automatic calibration system for micro-displacement devices is proposed. The automatic system according to the international specification of ASTM-E2309 has been developed. This system designed for the calibration of piezo transducers is based on the interferometric structure of the common optical path and possesses the resolution of the nanometer order. The experimental verifications demonstrate that the repeatability of the Fabry-Perot interferometer is less than $11 \mathrm{~nm}$. Experimental results of the synchronic measurement with the self-developed interferometer and a commercial interferometer reveal that the differences of the maximum nonlinearity error and maximum hysteresis error are less than $1 \%$. With the proposed correct equations, the maximum nonlinearity error can be minimized to $1 \%$ and the maximum hysteresis error will be less than $5.2 \%$.
\end{abstract}

Keywords - Fabry-Perot interferometer, Piezo transducer, Automatic calibration, Micro-displacement.

\section{INTRODUCTION}

With the development trends of high-performance, the requirements of processing accuracy are more and more demanding in the industry. The industries of MEMS, semiconductor and optic have the increasing demands for micro-actuators. Among the various types of actuators, only the piezo transducers can meet the requirement of the industries. Hence the calibration of the critical parameters for piezo transducers (PT) becomes more important to satisfy the applications in precision engineering.

The study according to the specification of ASTM-E2309 is to design an automatic calibration system for PT. The calibration system can not only reduce the risk of personal errors but also can be employed for the different specifications of PT. By selecting the appropriate measuring points and the measuring times, the nonlinearity error, hysteresis error and repeatability all can be calculated by the program automatically. 


\section{MEASUREMENT PRINCIPLE}

\subsection{Pizeo transducer}

The PT (the whole stroke: 22 micrometers) produced by Piezomechanik company is employed as a calibration object in this study. The converse piezoelectric effect will generate the displacement by drive voltages, and the critical parameters are measured by a selfdeveloped automatic calibration system.

\subsection{Measurement specification}

The study is based on the specification of the ASTM-E2309 which provided by the American Society for Materials and Testing to design the calibration system and operating procedure.

In accordance with the measurement procedure, at least five displacement values are required, and the each measurement interval should be no larger than one-third the difference in the whole stroke. And each cycle must be repeated two times. The sketch of measurement procedure is illustrated in Fig. 2-1.

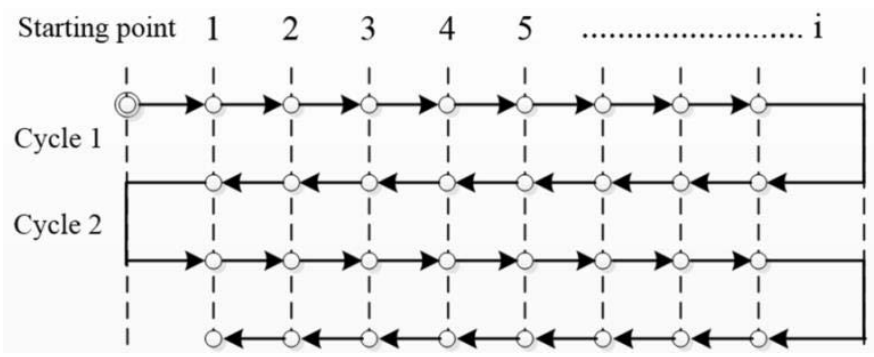

Fig. 2-1 Sketch of measurement procedure

\subsection{Fabry-Perot interferometer}

The PT possess the accuracy of nanometer-order and it can also displace within the range of sub-nanometer and nanometer. Previous investigations demonstrate that the laser interferometer is the appropriate calibration equipment to meet the demands.

The optical structure of the self-developed interferometer is demonstrated in the Fig. 2-2. Laser beam passes through the BS into the optical cavity. The one-eighth waveplate in the cavity is employed to form the orthogonal phase shift between interferometric signals. And the polarization axis of the waveplate must be the same as that of PBS. By this arrangement, the orthogonal signal can be acquired by two photodiodes (PDs) and then transmitted to the signal processing module.

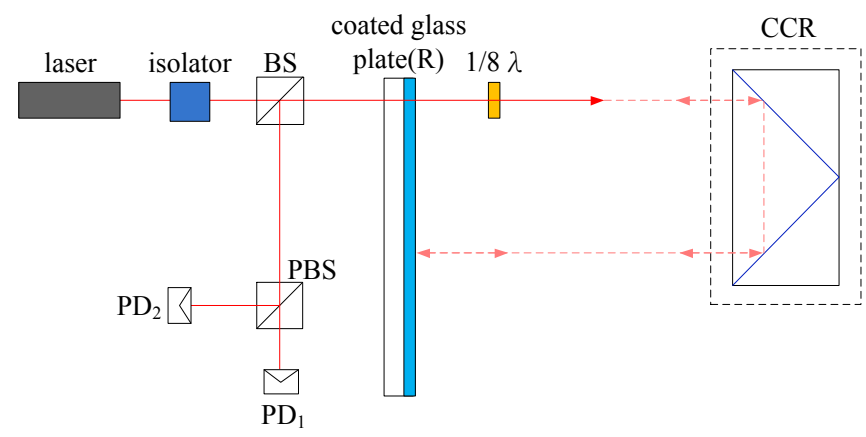

Fig. 2-2 Optical structure of the interferometer 
The corresponding equation of the folded Fabry-Perot interferometer can be derived as the followings. The amplitude of the firstly reflected beam is expressed as $A_{S 1}(2.1)$ and other interferometric beams can be denoted with $A_{S n}(2.2)$, where $A_{0}$ is the amplitude of incident light wave, $\mathrm{R}$ is the reflectivity of the coated glass plate, $\mathrm{L}$ is the resultant transmittance of the optical cavity, and $\mathrm{n}$ is the order number of the backward reflected light beam.

$$
\begin{gathered}
A_{S 1}=\frac{-1}{2 \sqrt{2}} A_{0} \sqrt{R} \\
A_{S n}=\frac{1}{2 \sqrt{2}} A_{0} T(L)^{n-1}(\sqrt{R})^{2 n-3}
\end{gathered}
$$

The corresponding electric field of the interferometric beam can be described as Eq. 2.3 and 2.4, where $\delta$ is the phase difference of the optical path. In this structure, $\delta$ is $\frac{4 \pi d}{\lambda}, \mathrm{d}$ is the distance between the planar mirror and CCR and $\omega$ is the angular frequency.

$$
\begin{gathered}
E_{S n}=\frac{1}{2 \sqrt{2}} A_{0} T(L)^{n-1}(\sqrt{R})^{2 n-3} e^{i(\omega t-k x+(2 n-2) \cdot \delta)} \\
E_{P n}=\frac{1}{2 \sqrt{2}} A_{0} T(L)^{n-1}(\sqrt{R})^{2 n-3} e^{i\left(\omega t-k x+(2 n-2) \cdot \delta-\frac{\pi}{2}\right)}
\end{gathered}
$$

By Eq. 2.5 and 2.6, the intensity distribution of s-type and p-type can be denoted with Eq. 2.7 and Eq. 2.8. And Fig. 2-3 illustrates the distribution of interferometric intensity by simulation.

$$
\begin{aligned}
& \mathrm{I}_{\mathrm{S}}=\mathrm{E}_{\mathrm{s}} \cdot \mathrm{E}_{\mathrm{s}}^{*} \\
& \mathrm{I}_{\mathrm{P}}=\mathrm{E}_{\mathrm{p}} \cdot \mathrm{E}_{\mathrm{p}}^{*}
\end{aligned}
$$

For s-type (PD1) intensity,

$$
\mathrm{I}_{\mathrm{S}}=\mathrm{E}_{\mathrm{S}} \cdot \mathrm{E}_{\mathrm{S}}^{*}=\frac{1}{8} \mathrm{~A}_{0}^{2} \frac{\mathrm{R}\left(1+\mathrm{L}^{2}\right)-2 \mathrm{LR} \cos (2 \delta)}{1+\mathrm{L}^{2} \mathrm{R}^{2}-2 \mathrm{LR} \cos (2 \delta)}
$$

For p-type (PD2) intensity,

$$
\mathrm{I}_{\mathrm{P}}=\mathrm{E}_{\mathrm{p}} \cdot \mathrm{E}_{\mathrm{p}}^{*}=\frac{1}{8} \mathrm{~A}_{0}{ }^{2} \frac{\mathrm{R}\left(1+\mathrm{L}^{2}\right)-2 \mathrm{LR} \cos \left(2 \delta-\frac{\pi}{2}\right)}{1+\mathrm{L}^{2} \mathrm{R}^{2}-2 \mathrm{LR} \cos \left(2 \delta-\frac{\pi}{2}\right)}
$$




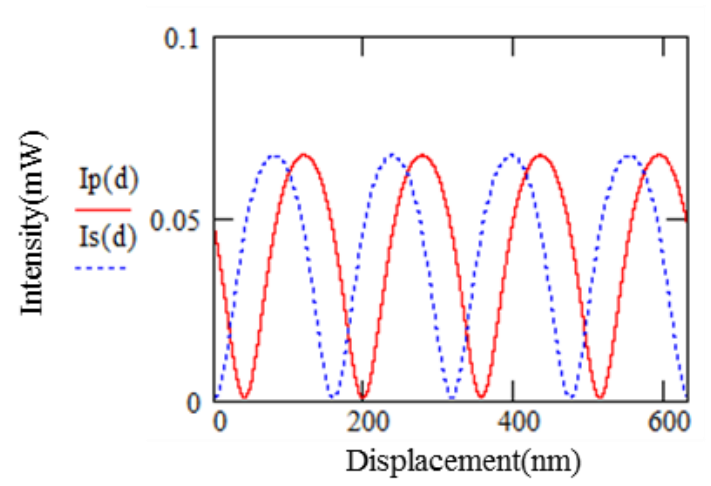

(a) Displacement vs. Intensity

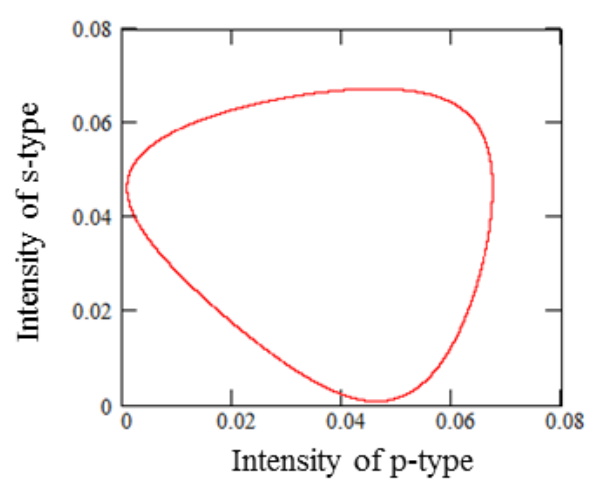

(b) s-p type

Fig. 2-3 Signal distribution by simulation

\section{THE DEVELOPED INTERFEROMETER SYSTEM}

\subsection{Optomechantronic system}

The previous study is based on the conventional structure of the Fabry-Perot interferometer as illustrated in Fig. 3-1. In the systematic structure, the signal offset or drift will occur. After the experimental verifications, the signal problem can be solved by the following modification. The planar mirror is replaced to the CCR and the sensor head is modified to the vertical mechanism.

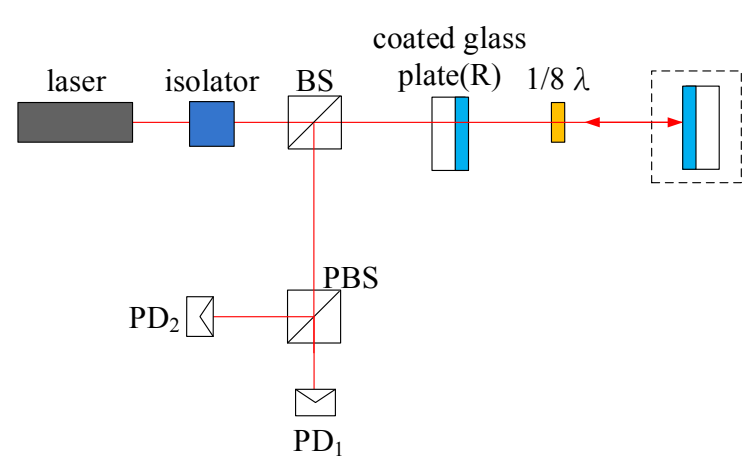

(a) Optical structure

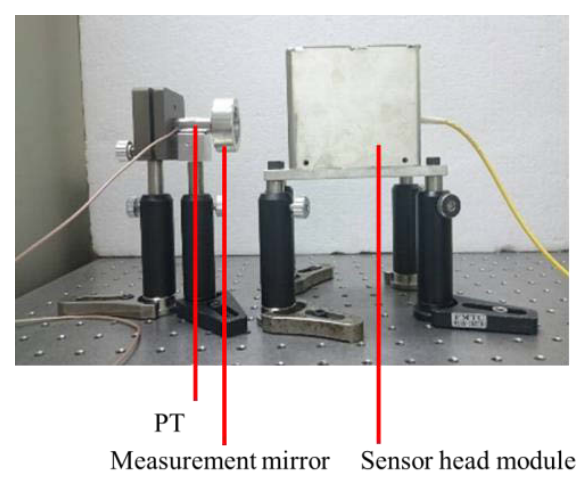

(b) Prototype of the sensor head

Fig. 3-1 Previous measurement structure

The modified structure of the Fabry-Perot interferometer is shown in Fig. 3-2. The system has three advantages as the following description, i.e. a closed system with a shell protection, by $\mathrm{CCR}$ as the measurement mirror to avoid the signal problem due to the tilt angle of the measurement mirror and more flexible to replace another calibration objects. 


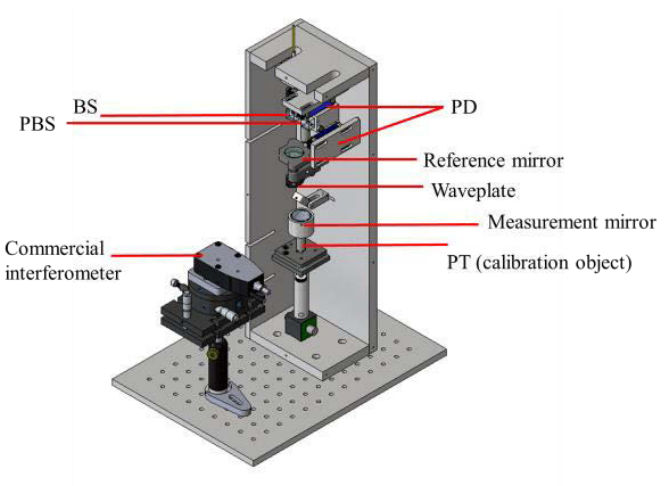

(a) Sketch of the system

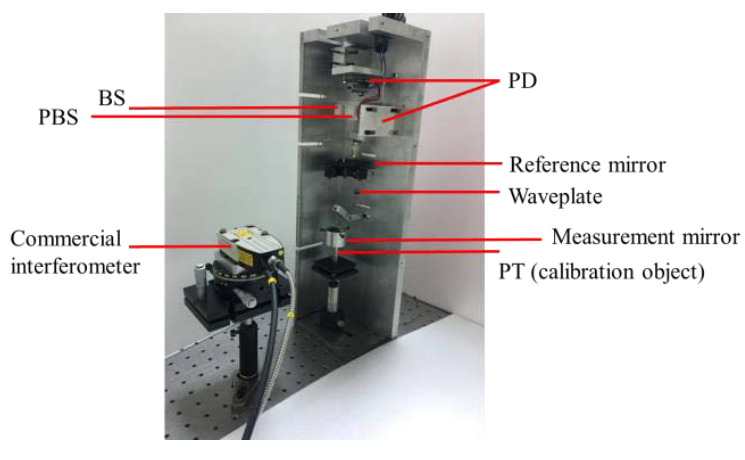

(b) Experiment setup

Fig. 3-2 Modified structure of the sensor head

\subsection{Signal processing module}

The signal reliability will directly influence with the measurement quality. However, the reliability can be assessed by the degree of the signal decline and DC offset. The excessive signal decline and DC offset is not allowed in the measurement process. The sketch of the signal decline and DC offset are demonstrated in Fig. 3-3 and Fig. 3-4.

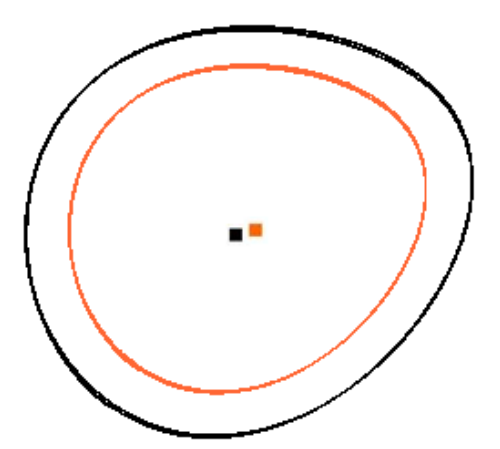

Fig. 3-3 Demonstration of the signal decline

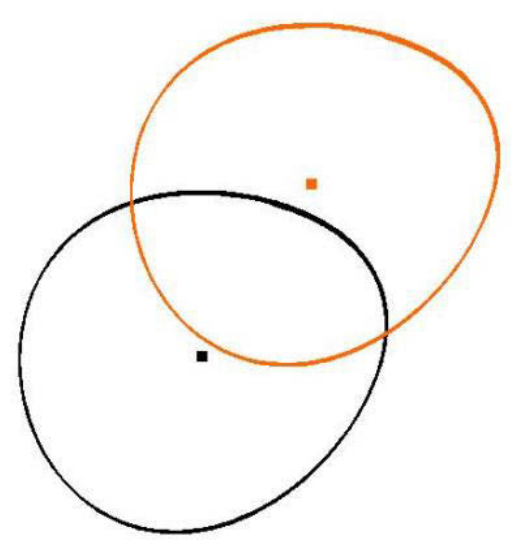

Fig. 3-4 Illustration of the DC offset

Therefore in order to improve the efficiency of the interferometric signal and the stability of the counting, the compensation module of auto gain control (AGC) and DC offset is developed in this study. The signal processing module is shown in Fig. 3-5.

The signal acquisition unit is realized by the DAQ board. By combining the quadrature 
encoders, the DAQ board and the program, the interferometric signal can be transformed into information of displacement. The other part of the signal processing module is the auto gain control (AGC) and DC offset compensation module. The orthogonal signal from the preamplifier is transmitted into the AGC and DC offset compensation module. Each signal has its different gain and offset control channels.

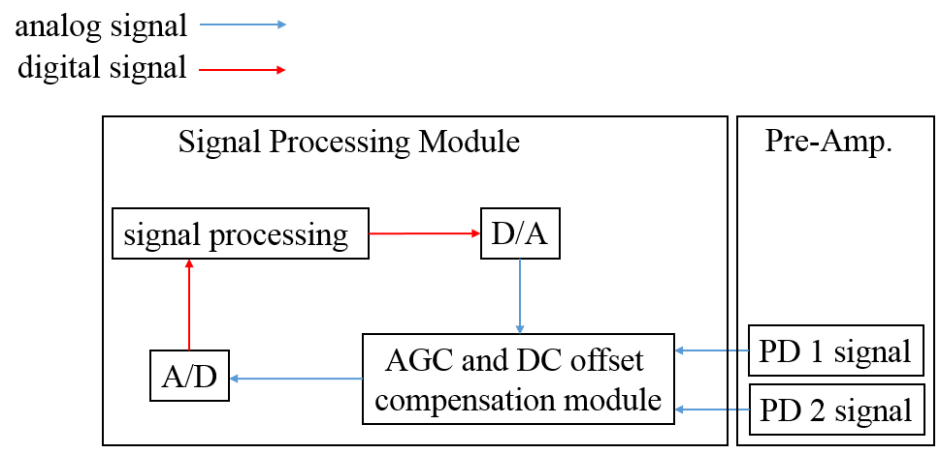

Fig. 3-5 Signal processing module

\subsection{Calibration system}

The core function is to develop a measurement program in accordance with the international specifications of ASTM-E2309. Users only need to enter the appropriate measuring points and the measuring times, the critical parameters can all be calculated by the program automatically. The structure of the calibration system is illustrated in Fig. 3-6.

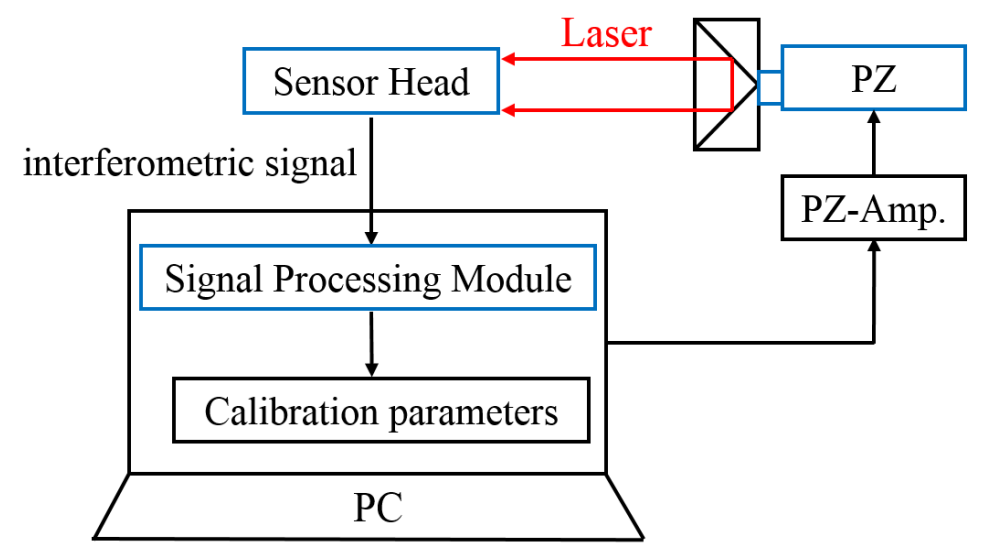

Fig. 3-6 Structure of the calibration system

\section{EXPERIMENTAL RESULTS AND ANALYSIS}

\subsection{Measurement results}

The whole stroke of the PT is divided into six measuring points and each cycle is repeated three times. In the experiment, the measurement results of the F-P interferometer are compared with the results of the Renishaw RLE10 interferometer.

With the step interval of $30 \mathrm{~V}$, the drive voltage is altered from $0 \mathrm{~V}$ to $150 \mathrm{~V}$ and at each position 30 data will be acquired and then averaged. Drive voltages versus the displacement (V-D curve) for the PT with three measuring cycles is presented in Fig. 4-1. The measurement results reveal that there is a hysteresis deviation between the forward direction and the backward direction. 


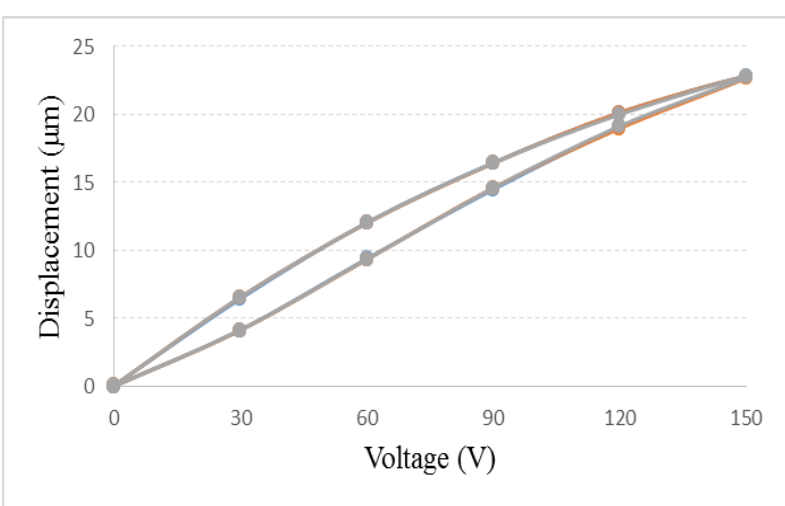

(a) The F-P interferometer

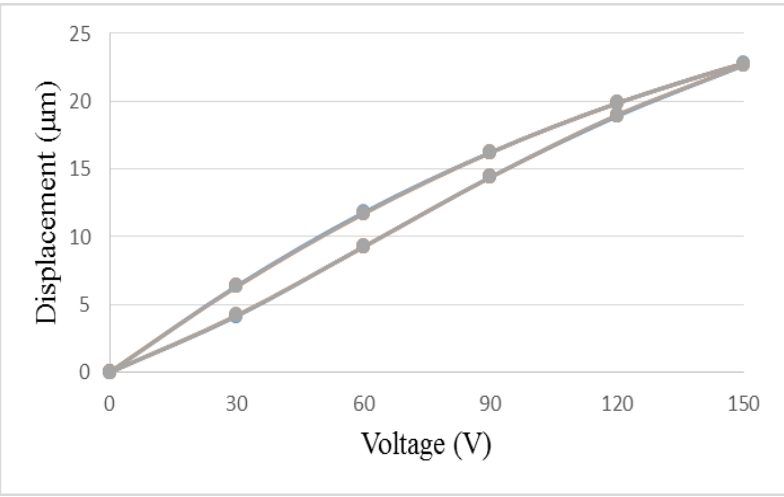

(b) The Renishaw RLE10 interferometer

Fig. 4-1 Drive voltages vs. Displacement

\subsubsection{Maximum nonlinearity error}

From the Fig. 4-1, the V-D curves of the three measuring cycles are averaged, and then an averaged V-D curve can be obtained. To represent the nonlinearity error, the differences between the measured curve and the fitted curve are divided by the full stroke and are denoted with the percentage. The experimental results reveal that the maximum nonlinearity error of the Fabry-Perot interferometer in forward direction is $2.6 \%$, and the maximum nonlinearity error of the Renishaw RLE10 interferometer in forward direction is $2.3 \%$ as shown in Fig. 4-2.

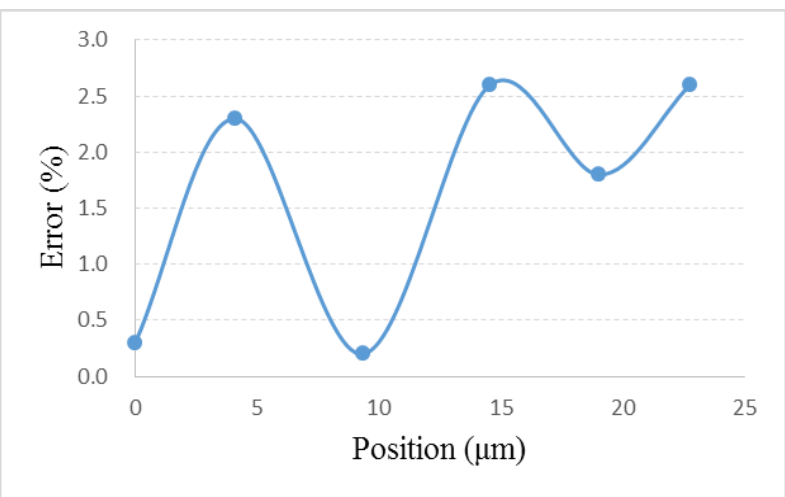

(a) The F-P interferometer

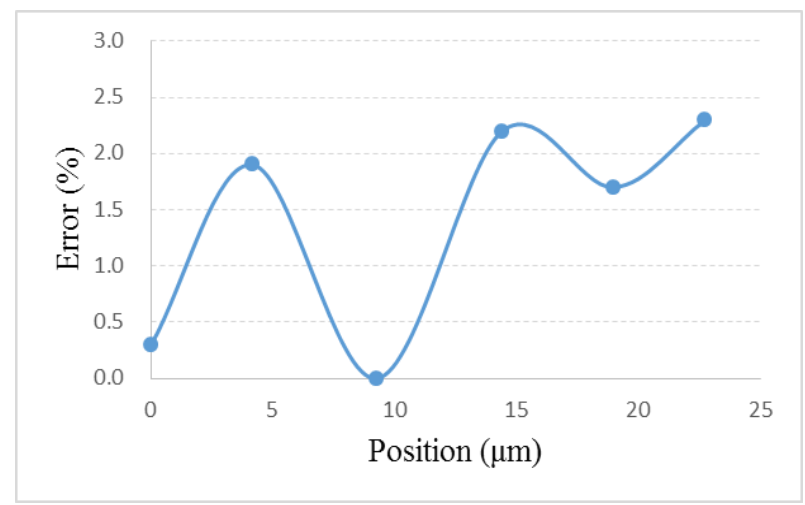

(b) The Renishaw RLE10 interferometer

Fig. 4-2 Nonlinearity error in forward direction

The maximum nonlinearity error of the Fabry-Perot interferometer in backward direction is $7.3 \%$, and the maximum nonlinearity error of the Renishaw RLE10 interferometer in backward direction is $6.7 \%$ as illustrated in Fig. 4-3. 


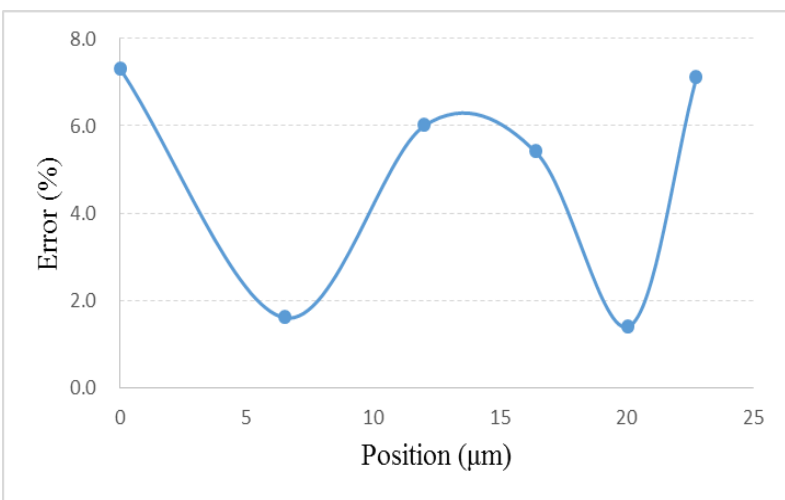

(a) The F-P interferometer

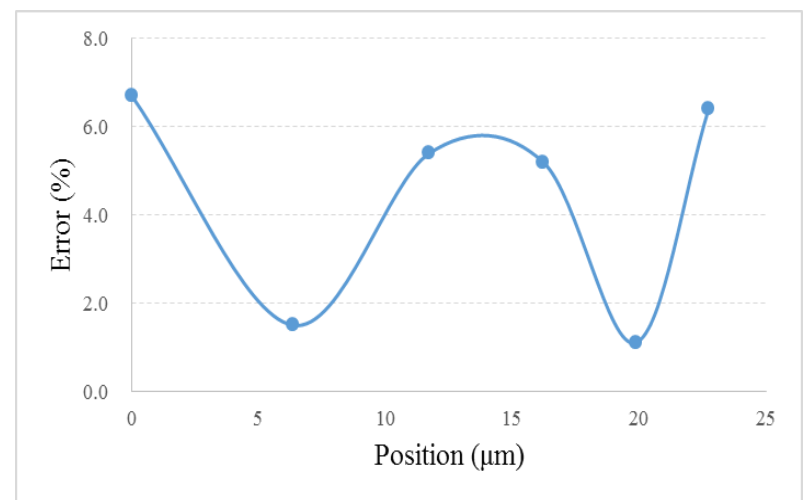

(b) The Renishaw RLE10 interferometer

Fig. 4-3 Nonlinearity error in backward direction

\subsubsection{Maximum hysteresis error}

According to the averaged V-D curve, the differences between the forward and the backward displacement under the same drive voltage are divided by the full stroke and are denoted with the percentage to represent the hysteresis error. The results reveal that the maximum hysteresis error of the Fabry-Perot interferometer is $12.1 \%$; the maximum hysteresis error of the Renishaw RLE10 interferometer is $11.3 \%$ as demonstrated in Fig. 4-4.

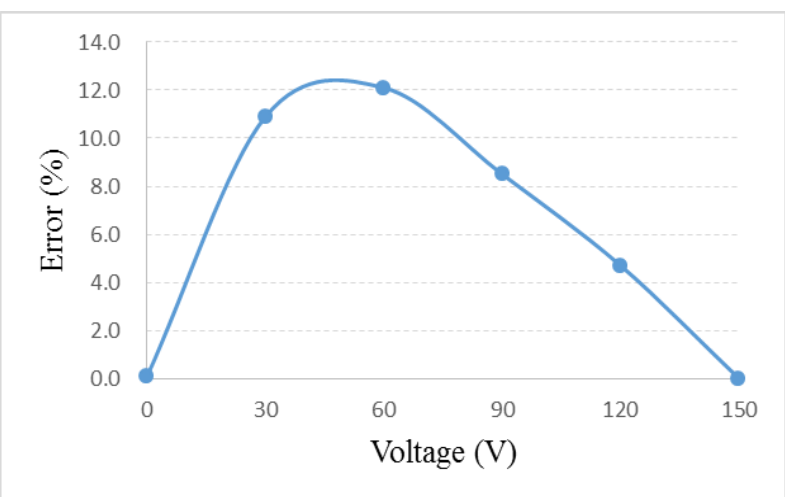

(a) The F-P interferometer

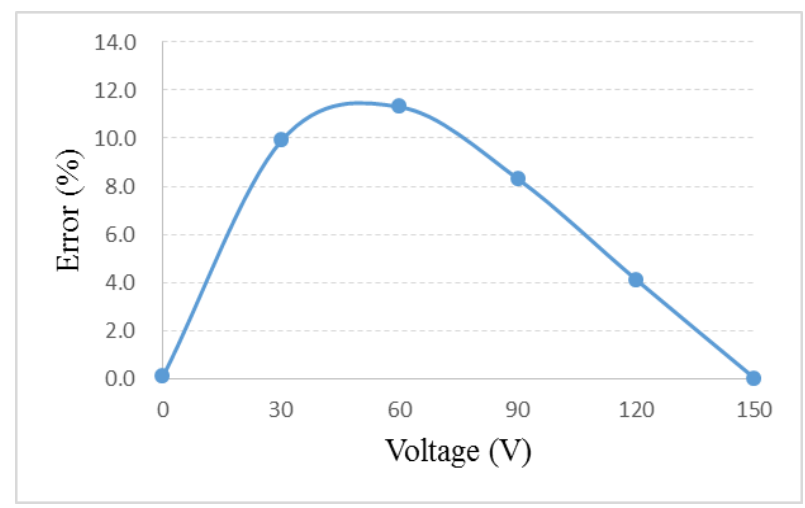

(b) The Renishaw RLE10 interferometer

Fig. 4-4 Hysteresis error

\subsubsection{Repeatability}

In the experiment, the standard deviation of each position can be obtained by calculating 30 measured data under the different drive voltage. The results reveal that the repeatability of the Fabry-Perot interferometer is less than $0.011 \mu \mathrm{m}$ as shown in Fig. 4-5. 


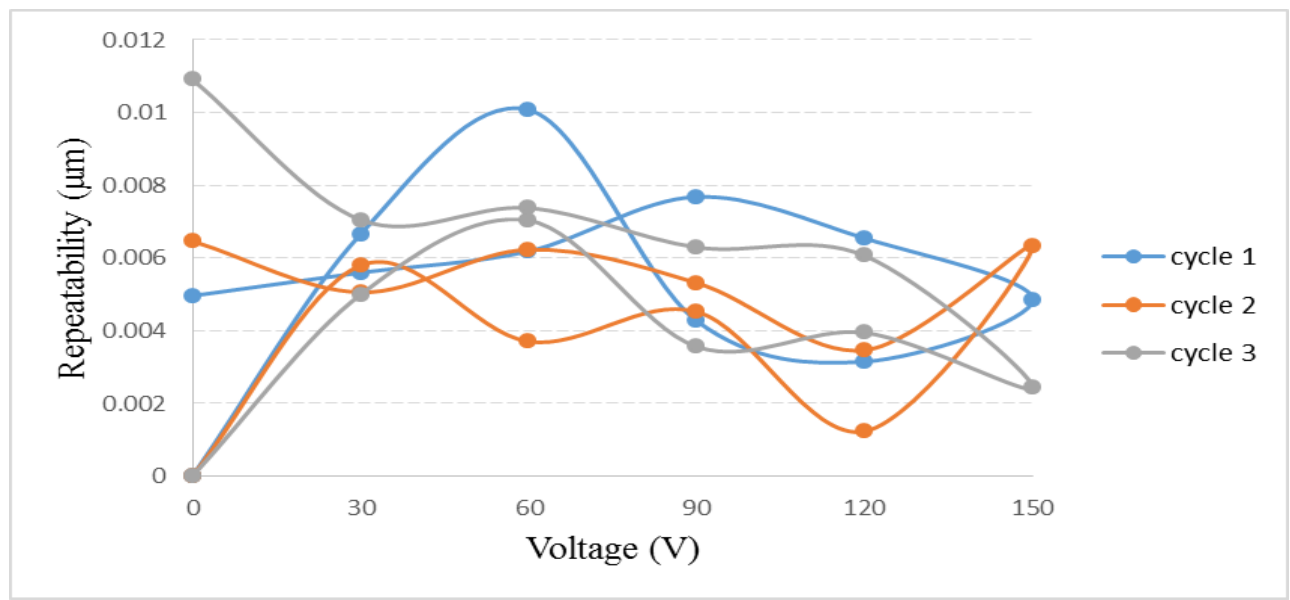

Fig. 4-5 Repeatability of each measuring point

From the experimental results, the comparison results between Fabry-Perot interferometer and Renishaw interferometer are presented as the following Table 4-1.

Table 4-1 Comparison results

\begin{tabular}{|c|c|c|c|c|}
\hline Interferometer & \multicolumn{2}{|c|}{ Fabry-Perot } & \multicolumn{2}{c|}{ Renishaw RLE-10 } \\
\hline Direction & forward & backward & forward & backward \\
\hline Maximum nonlinearity error & $2.6 \%$ & $7.3 \%$ & $2.3 \%$ & $6.7 \%$ \\
\hline Maximum hysteresis error & \multicolumn{2}{|c|}{$12.1 \%$} & \multicolumn{2}{c|}{$11.3 \%$} \\
\hline
\end{tabular}

\subsection{Calibration results}

Because the repeatability of the Fabry-Perot interferometer developed in this study is less than $0.011 \mu \mathrm{m}$, the fourth order polynomial $\left(S=a v^{4}+b v^{3}+c v^{2}+d v+e\right)$ fitted method can be utilized to fit the forward and backward curves respectively. By calculating the inverse function of the equation, the corresponding drive voltage of the desired position can be obtained.

The fourth order fitted equation in forward direction $S_{c}$ :

$$
S_{c}=3 E-08 v^{4}-1 E-05 v^{3}+0.0016 v^{2}+0.1029 v+0.0008
$$

The fourth order fitted equation in backward direction $S_{b}$ :

$$
S_{b}=-4 E-09 v^{4}+2 E-06 v^{3}-0.0007 v^{2}+0.2407 v-0.0227
$$

The inverse function in forward direction $f^{-1}\left(\mathrm{~S}_{\mathrm{c}}\right)$ :

$$
f^{-1}\left(\mathrm{~S}_{\mathrm{c}}\right)=-0.0002 \mathrm{~S}_{\mathrm{c}}{ }^{4}+0.0143 \mathrm{~S}_{\mathrm{c}}{ }^{3}-0.3268 \mathrm{~S}_{\mathrm{c}}{ }^{2}+8.2809 \mathrm{~S}_{\mathrm{c}}+0.016
$$

The inverse function in backward direction $f^{-1}\left(\mathrm{~S}_{\mathrm{b}}\right)$ :

$$
f^{-1}\left(\mathrm{~S}_{\mathrm{b}}\right)=0.0004 \mathrm{~S}_{\mathrm{b}}{ }^{4}-0.0114 \mathrm{~S}_{\mathrm{b}}{ }^{3}+0.1915 \mathrm{~S}_{\mathrm{b}}{ }^{2}+3.6572 \mathrm{~S}_{\mathrm{b}}-0.0822
$$


With the step interval of $4.4 \mu \mathrm{m}$, the desired position is altered from $0 \mu \mathrm{m}$ to $22 \mu \mathrm{m}$ and at each position 30 data will be acquired and then averaged. Position versus the displacement (P-D curve) for the PT is presented in Fig. 4-6. Obviously, the linearity has greatly improved.
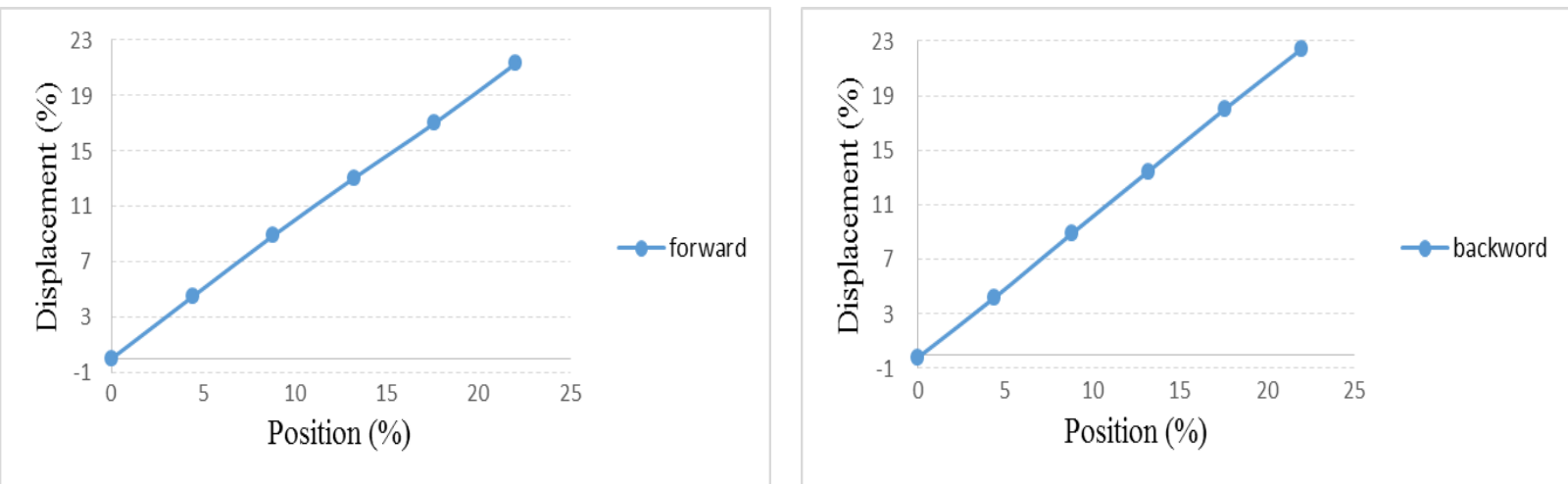

Fig. 4-6 Position vs. Displacement

\subsubsection{Maximum nonlinearity error}

From the Fig. 4-6, the P-D curves of the three measuring cycles are averaged, and then an averaged P-D curve can be obtained. To represent the nonlinearity error, the differences between the measured curve and the fitted curve are divided by the full stroke and are denoted with the percentage. The results of the Fabry-Perot interferometer reveal that the maximum nonlinearity error in forward direction is $1 \%$; the maximum nonlinearity error in backward direction is $0.5 \%$ as shown in Fig. $4-7$.
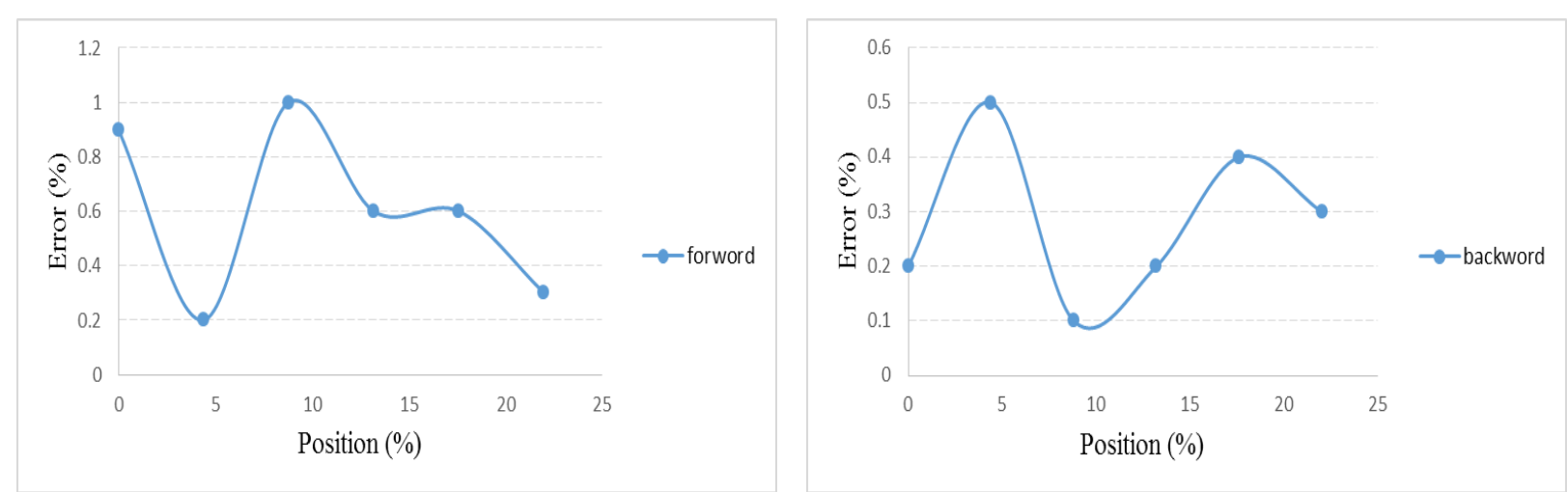

Fig. 4-7 Nonlinearity error

\subsubsection{Maximum hysteresis error}

With the averaged P-D curve, the difference between the forward and the backward displacement under the same position are divided by the full stroke and are denoted with the percentage to represent the hysteresis error. The measurement results of the Fabry-Perot interferometer reveal that the maximum hysteresis error is $5.2 \%$ as illustrated in Fig. 4-8. 


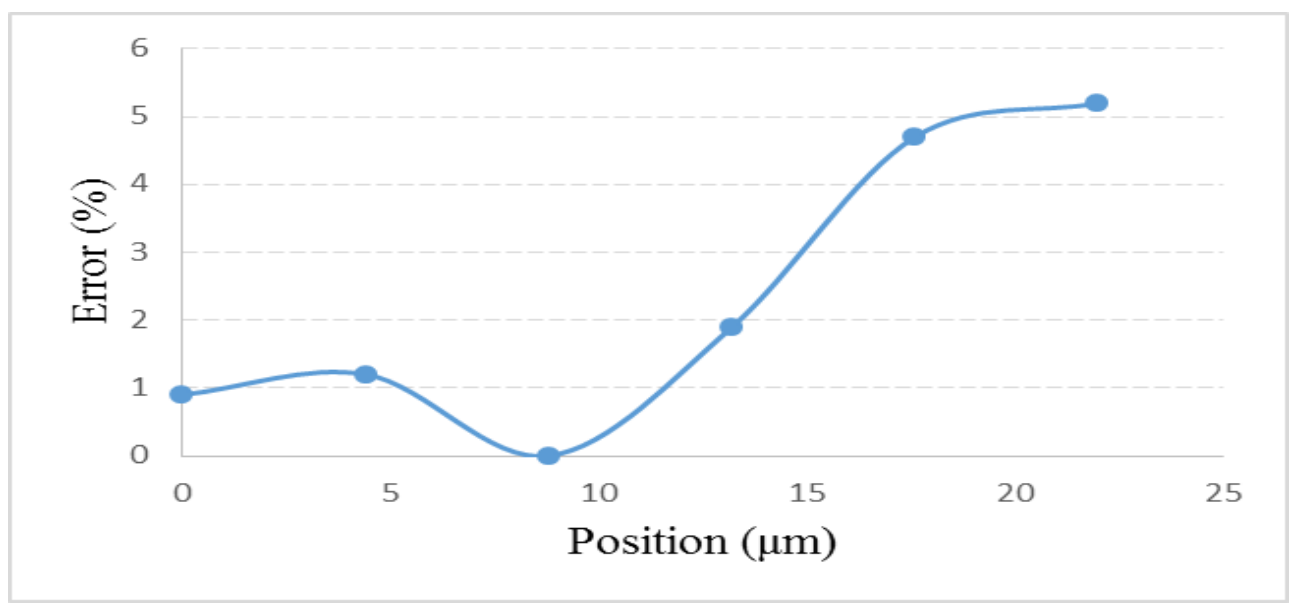

Fig. 4-8 Hysteresis error

According to the experimental results shown in Fig. 4-6 to 4-8, the linearity of the system has been improved after the calibration procedure. The comparison results are presented as the following Table 4-2.

Table 4-2 Comparison with the calibration results

\begin{tabular}{|c|c|c|c|c|}
\hline Fabry-Perot interferometer & \multicolumn{2}{|c|}{ Without calibration } & \multicolumn{2}{c|}{ With Calibration } \\
\hline Direction & forward & backward & forward & backward \\
\hline Maximum non-linearity error & $2.6 \%$ & $7.3 \%$ & $1 \%$ & $0.5 \%$ \\
\hline Maximum hysteresis error & \multicolumn{2}{|c|}{$12.1 \%$} & \multicolumn{2}{c|}{$5.2 \%$} \\
\hline
\end{tabular}

\section{CONCLUSION}

In this investigation, an automatic calibration system for the PT has been proposed. With the experimental verifications of the repeatability, the Fabry-Perot interferometer possesses the high precision. Comparison of measurement results between the self-developed interferometer and the commercial interferometer reveal that the differences of the maximum nonlinearity error and maximum hysteresis error are less than $1 \%$. After the calibration procedure, the maximum nonlinearity error can be improved to $1 \%$ and the maximum hysteresis error will be reduced to $5.2 \%$.

\section{REFERENCES}

[1] Attilio Sacconi, Gian Bartolo Picotto, and Walter Pasin, "The IMGC Calibration Setup",IEEE Transactions on Instrumentation and Measurement, Vol. 48, NO. 2, pp.385386, April 1999.

[2] Luigi Bruno, Paolo Mainieri, and Andrea Poggialini, "Design and calibration of a lowcost open-loop PZT actuator for phase-shifting speckle interferometry", Proc. of SPIE Vol. 4933, pp.317-322, Speckle Metrology 2003.

[3] Luigi Bruno, Andrea Poggialini and Giuseppina Felice, "Design and calibration of a piezoelectric actuator for interferometric applications", Optics and Lasers in Engineering, Volume 45, Issue 12, pp.1148-1156, December 2007. 
[4] Francisco de Assis Andrade Barbosa, Gilder Nader, Ricardo Tokio Higuti, Cláudio Kitano, "A Simple Interferometric Method To Measure The Calibration Factor And Displacement Amplification In Piezoelectric Flextensional Actuators", Revista Controle \& Automação, Vol. 21, No.6, pp.577-587, Novembro e Dezembro 2010.

[5] International Standard: ASTM-E2309, "Standard Practices for Verification of Displacement Measuring Systems and Devices Used in Material Testing Machines", Reapproved 2011.

\section{CONTACTS}

Hung-Ta Shih

citizen201322@gmail.com

Yung-Cheng Wang

wangyc@yuntech.edu.tw

Lih-Horng Shyu

lhshyu@nfu.edu.tw 